\title{
Cyclic $(\phi)$-Contractions in Uniform Spaces and Related Fixed Point Results
}

\author{
N. Hussain, ${ }^{1}$ E. Karapınar, ${ }^{2}$ S. Sedghi, ${ }^{3}$ N. Shobkolaei, ${ }^{4}$ and S. Firouzian ${ }^{5}$ \\ ${ }^{1}$ Department of Mathematics, King Abdulaziz University, P.O. Box 80203, Jeddah 21589, Saudi Arabia \\ ${ }^{2}$ Department of Mathematics, Atilim University, Incek, 06836 Ankara, Turkey \\ ${ }^{3}$ Department of Mathematics, Qaemshahr Branch, Islamic Azad University, Qaemshahr, Iran \\ ${ }^{4}$ Department of Mathematics, Babol Branch, Islamic Azad University, Babol, Iran \\ ${ }^{5}$ Payame Noor University, Babol, Iran
}

Correspondence should be addressed to N. Hussain; nhusain@kau.edu.sa

Received 25 December 2013; Accepted 30 January 2014; Published 11 March 2014

Academic Editor: Chi-Ming Chen

Copyright (C) 2014 N. Hussain et al. This is an open access article distributed under the Creative Commons Attribution License, which permits unrestricted use, distribution, and reproduction in any medium, provided the original work is properly cited.

First, we define cyclic $(\phi)$-contractions of different types in a uniform space. Then, we apply these concepts of cyclic $(\phi)$-contractions to establish certain fixed and common point theorems on a Hausdorff uniform space. Some more general results are obtained as corollaries. Moreover, some examples are provided to demonstrate the usability of the proved theorems.

\section{Introduction}

Let $X$ be a nonempty set. A nonempty family, $\vartheta$, of subsets of $X \times X$ is called the uniform structure of $X$ if it satisfies the following properties:

(i) if $G$ is in $\vartheta$, then $G$ contains the diagonal $\{(x, x) \mid x \in$ $X$;

(ii) if $G$ is in $\vartheta$ and $H$ is a subset of $X \times X$ which contains $G$, then $H$ is in $\vartheta$;

(iii) if $G$ and $H$ are in $\vartheta$, then $G \cap H$ is in $\vartheta$;

(iv) if $G$ is in $\mathcal{\vartheta}$, then there exists $H$ in $\mathcal{\vartheta}$, such that, whenever $(x, y)$ and $(y, z)$ are in $H$, then $(x, z)$ is in G;

(v) if $G$ is in $\vartheta$, then $\{(y, x) \mid(x, y) \in G\}$ is also in $\vartheta$.

The pair $(X, 9)$ is called a uniform space and the element of $\vartheta$ is called entourage or neighbourhood or surrounding. The pair $(X, \vartheta)$ is called a quasi-uniform space (see, e.g., $[1,2])$ if property (v) is omitted.

Existence and uniqueness of fixed points for various contractive mappings in the setting of uniform spaces have been investigated by several authors; see, for example, [3-12] and the references therein.
Recently, an interesting and remarkable notion of cyclic mapping was introduced and studied by Kirk et al. [13]. Following this paper, a number of authors introduced contractive mapping via the cyclic mappings and reported certain fixed point results in the setting of different type of spaces; see, for example, [13-17].

In this paper, we will give the characterization of cyclic mapping in the context of uniform spaces and, further, prove the existence and uniqueness of fixed and common fixed points of such mappings via $A$-distance and $E$-distance, introduced by Aamri and El Moutawakil [18].

For the sake of completeness, we recollect some basic definitions and fundamental results. Let $\Delta=\{(x, x) \mid x \in X\}$ be the diagonal of a nonempty set $X$. For $V, W \in X \times X$, we will use the following setting in the sequel:

$$
\begin{aligned}
& V \circ W \\
& =\{(x, y) \mid \text { there exists } z \in X:(x, z) \in W,(z, y) \in V\}, \\
& V^{-1}=\{(x, y) \mid(y, x) \in V\} .
\end{aligned}
$$

For subset $V \in \mathcal{Y}$, a pair of points $x$ and $y$ are said to be $V$ close if $(x, y) \in V$ and $(y, x) \in V$. Moreover, a sequence $\left\{x_{n}\right\}$ 
in $X$ is called a Cauchy sequence for $\vartheta$, if for any $V \in \vartheta$ there exists $N \geq 1$ such that $x_{n}$ and $x_{m}$ are $V$-close for $n, m \geq N$. For $(X, \vartheta)$, there is a unique topology $\tau(\vartheta)$ on $X$ generated by $V(x)=\{y \in X \mid(x, y) \in V\}$, where $V \in \mathcal{\vartheta}$.

A sequence $\left\{x_{n}\right\}$ in $X$ is convergent to $x$ for $\vartheta$, denoted by $\lim _{n \rightarrow \infty} x_{n}=x$, if for any $V \in \mathcal{\vartheta}$ there exists $n_{0} \in \mathbb{N}$ such that $x_{n} \in V(x)$ for every $n \geq n_{0}$. A uniform space $(X, \vartheta)$ is called Hausdorff if the intersection of all the $V \in \mathcal{\vartheta}$ is equal to $\Delta$ of $X$, that is, if $(x, y) \in V$ for all $V \in \vartheta$ implies $x=y$. If $V=V^{-1}$, then we say that a subset $V \in \mathcal{Y}$ is symmetrical. Throughout the paper, we assume that each $V \in \mathcal{\vartheta}$ is symmetrical. For more details, see, for example, [1, 18-21].

Now, we recall the notions of $A$-distance and $E$-distance.

Definition 1 (see, e.g., $[18,19])$. Let $(X, \vartheta)$ be a uniform space. A function $p: X \times X \rightarrow[0, \infty)$ is said to be an $A$-distance if for any $V \in \mathcal{\vartheta}$ there exists $\delta>0$ such that if $p(z, x) \leq \delta$ and $p(z, y) \leq \delta$ for some $z \in X$, then $(x, y) \in V$.

Definition 2 (see, e.g., $[18,19])$. Let $(X, \vartheta)$ be a uniform space. A function $p: X \times X \rightarrow[0, \infty)$ is said to be an $E$-distance if

$\left(p_{1}\right) p$ is an $A$-distance,

$$
\left(p_{2}\right) p(x, y) \leq p(x, z)+p(z, y), \forall x, y, z \in X \text {. }
$$

Example 3 (see, e.g., $[18,19])$. Let $(X, \vartheta)$ be a uniform space and let $d$ be a metric on $X$. It is evident that $\left(X, \vartheta_{d}\right)$ is a uniform space, where $\vartheta_{d}$ is the set of all subsets of $X \times X$ containing a "band" $B_{\epsilon}=\left\{(x, y) \in X^{2} \mid d(x, y)<\epsilon\right\}$ for some $\epsilon>0$. Moreover, if $\vartheta \subseteq \vartheta_{d}$, then $d$ is an $E$-distance on $(X, \vartheta)$.

Lemma 4 (see, e.g., $[18,19])$. Let $(X, 9)$ be a Hausdorff uniform space and let $p$ be an A-distance on X. Let $\left\{x_{n}\right\}$ and $\left\{y_{n}\right\}$ be sequences in $X$ and $\left\{\alpha_{n}\right\}$ and let $\left\{\beta_{n}\right\}$ be sequences in $[0, \infty)$ converging to 0 . Then, for $x, y, z \in X$, the following hold.

(a) If $p\left(x_{n}, y\right) \leq \alpha_{n}$ and $p\left(x_{n}, z\right) \leq \beta_{n}$ for all $n \in \mathbb{N}$, then $y=z$. In particular, if $p(x, y)=0$ and $p(x, z)=0$, then $y=z$.

(b) If $p\left(x_{n}, y_{n}\right) \leq \alpha_{n}$ and $p\left(x_{n}, z\right) \leq \beta_{n}$ for all $n \in \mathbb{N}$, then $\left\{y_{n}\right\}$ converges to $z$.

(c) If $p\left(x_{n}, x_{m}\right) \leq \alpha_{n}$ for all $n, m \in \mathbb{N}$ with $m>n$, then $\left\{x_{n}\right\}$ is a Cauchy sequence in $(X, \vartheta)$.

Let $p$ be an $A$-distance. A sequence in a uniform space $(X, \vartheta)$ with an $A$-distance is said to be a $p$-Cauchy if for every $\epsilon>0$ there exists $n_{0} \in \mathbb{N}$ such that $p\left(x_{n}, x_{m}\right)<\epsilon$ for all $n, m \geq n_{0}$.

Definition 5 (see, e.g., $[18,19])$. Let $(X, \vartheta)$ be a uniform space and let $p$ be an $A$-distance on $X$.

(1) $X$ is $S$-complete if for every $p$-Cauchy sequence $\left\{x_{n}\right\}$ there exists $x$ in $X$ with $\lim _{n \rightarrow \infty} p\left(x_{n}, x\right)=0$.

(2) $X$ is $p$-Cauchy complete if for every $p$-Cauchy sequence $\left\{x_{n}\right\}$ there exists $x$ in $X$ with $\lim _{n \rightarrow \infty} x_{n}=x$ with respect to $\tau(\vartheta)$.
Remark 6. Let $(X, \vartheta)$ be a Hausdorff uniform space which is $S$-complete. If a sequence $\left\{x_{n}\right\}$ is a $p$-Cauchy sequence, then we have $\lim _{n \rightarrow \infty} p\left(x_{n}, x\right)=0$. Regarding Lemma 4(b), we derive that $\lim _{n \rightarrow \infty} x_{n}=x$ with respect to the topology $\tau(\vartheta)$ and hence $S$-completeness implies $p$-Cauchy completeness.

Definition 7 . Let $(X, \vartheta)$ be a Hausdorff uniform space and let $p$ be an $A$-distance on $X$. Two self-mappings $f$ and $g$ of $X$ are said to be weak compatible if they commute at their coincidence points; that is, $f x=g x$ implies that $f g x=g f x$.

We denote by $\mathscr{F}$ the class of functions $\phi:[0, \infty) \rightarrow$ $[0, \infty)$ nondecreasing and continuous satisfying $\phi(t)>0$ for $t \in(0, \infty)$ and $\phi(0)=0$.

Definition 8 (see [17]). A function $\phi:[0, \infty) \rightarrow[0, \infty)$ is called a comparison function if it satisfies the following:

(i) $\phi$ is increasing; that is, $t_{1} \leq t_{2}$ implies $\phi\left(t_{1}\right) \leq \phi\left(t_{2}\right)$, for $t_{1}, t_{2} \in[0, \infty)$;

(ii) $\left\{\phi^{n}(t)\right\}_{n \in \mathbb{N}}$ converges to 0 as $n \rightarrow \infty$, for all $t \in$ $[0, \infty)$.

Definition 9 (see [22]). A function $\phi:[0, \infty) \rightarrow[0, \infty)$ is called a (c)-comparison function if

(i) $\phi$ is increasing,

(ii) there exist $k_{0} \in \mathbb{N}, a \in(0,1)$ and a convergent series of nonnegative terms $\sum_{k=1}^{\infty} v_{k}$ such that

$$
\phi^{k+1}(t) \leq a \phi^{k}(t)+v_{k}
$$

for $k \geq k_{0}$ and any $t \in[0, \infty)$.

Let $\mathscr{C}$ be the collection of all (c)-comparison functions $\phi:[0, \infty) \rightarrow[0, \infty)$ defined in Definition 9 .

Lemma 10 (see [22]). If $\phi:[0, \infty) \rightarrow[0, \infty)$ is a (c)comparison function, then the following hold:

(i) $\phi$ is comparison function,

(ii) $\phi(t)<t$, for any $t \in[0, \infty)$,

(iii) $\phi$ is continuous at 0 ,

(iv) the series $\sum_{k=0}^{\infty} \phi^{k}(t)$ converges for any $t \in[0, \infty)$.

In 1922, Banach proved that every contraction in a complete metric space has a unique fixed point. This celebrated result has been generalized and improved by many authors in the context of different abstract spaces for various operators (see [1-28] and the references therein). Recently, fixed point theorems for operators $T$ defined on a complete metric space $X$ with a cyclic representation of $X$ with respect to $T$ have appeared in the literature (see, e.g., [13-17]). Now, we present a modification of the main result of [16]. For this, we need the following definitions.

Definition 11 (see [13]). Let $X$ be a nonempty set, $m$ a positive integer, and $T: X \rightarrow X$ a mapping. $X=\bigcup_{i=1}^{m} A_{i}$ is said to be a cyclic representation of $X$ with respect to $T$ if 
(i) $A_{i}, i=1,2, \ldots, m$, are nonempty sets;

(ii) $T\left(A_{1}\right) \subset A_{2}, \ldots, T\left(A_{m-1}\right) \subset A_{m}, T\left(A_{m}\right) \subset A_{1}$.

Definition 12. Let $(X, d)$ be a metric space, $m$ a positive integer, $A_{1}, A_{2}, \ldots, A_{m}$ nonempty subsets of $X$, and $X=$ $\bigcup_{i=1}^{m} A_{i}$. An operator $T: X \rightarrow X$ is a cyclic $(\phi)$-contraction if

(i) $X=\bigcup_{i=1}^{m} A_{i}$ is a cyclic representation of $X$ with respect to $T$,

(ii) $d(T x, T y) \leq \phi(d(x, y))$, for any $x \in A_{i}, y \in A_{i+1}$, $i=1,2, \ldots, m$, where $A_{m+1}=A_{1}$ and $\phi \in \mathscr{F}$.

The main result of [14] is the following.

Theorem 13 (Theorem 6 of [14]). Let $(X, d)$ be a complete metric space, $m$ a positive integer, $A_{1}, A_{2}, \ldots, A_{m}$ nonempty subsets of $X$, and $X=\bigcup_{i=1}^{m} A_{i}$. Let $T: X \rightarrow X$ be a cyclic $(\phi-\psi)$-contraction with $\phi, \psi \in \mathscr{F}$. Then, $T$ has a unique fixed point $z \in \bigcap_{i=1}^{m} A_{i}$.

The main aim of this paper is to prove results similar to the abovementioned theorems in uniform spaces and to present modifications of Theorem 2.1 [16], Theorems 3.1-3.2 in [18], and other related results.

\section{Main Result}

First, we present the following definition.

Definition 14. Let $(X, \vartheta)$ be a uniform space, $m$ a positive integer, $A_{1}, A_{2}, \ldots, A_{m}$ nonempty subsets of $X$, and $X=$ $\bigcup_{i=1}^{m} A_{i}$. An operator $T: X \rightarrow X$ is a cyclic $(\phi)$-contraction if

(i) $X=\bigcup_{i=1}^{m} A_{i}$ is a cyclic representation of $X$ with respect to $T$,

(ii) for any $x \in A_{i}, y \in A_{i+1}, i=1,2, \ldots, m$,

$$
p(T x, T y) \leq \phi(p(x, y))
$$

where $A_{m+1}=A_{1}$ and $\phi \in \mathscr{C}$.

Our main result is the following.

Theorem 15. Let $(X, \vartheta)$ be an S-complete Hausdorff uniform space such that $p$ is an E-distance on $X, m$ a positive integer, and $A_{1}, A_{2}, \ldots, A_{m}$ nonempty closed subsets of $X$ with respect to the topological space $(X, \tau(\vartheta))$, and $X=\bigcup_{i=1}^{m} A_{i}$. Let $T$ : $X \rightarrow X$ be a cyclic $(\phi)$-contraction. Then, T has a unique fixed point $x \in \bigcap_{i=1}^{m} A_{i}$

Proof. We first show that the fixed point of $T$ is unique (if it exists). Suppose, on the contrary, that $y, z \in X$ with $y \neq z$ are fixed points of $T$. The cyclic character of $T$ and the fact that $y, z \in X$ are fixed points of $T$ imply that $y, z \in \bigcap_{i=1}^{m} A_{i}$. Using the contractive condition, we obtain

$$
p(y, z)=p(T y, T z) \leq \phi(p(y, z))<p(y, z)
$$

and from the last inequality

$$
p(y, z)=0 .
$$

Similarly, we can show that $p(y, y)=0$ and, consequently, $y=z$.

Now, we prove the existence of a fixed point. Note that $p$ is not symmetric. To show that the sequence $\left\{x_{n}\right\}$ is Cauchy, we will show that both $\lim _{n \rightarrow \infty} p\left(x_{n}, x_{n+q}\right)=0$ and $\lim _{n \rightarrow \infty} p\left(x_{n+q}, x_{n}\right)=0$, for any $q>1$. by

For this aim, take $x_{0} \in X$ and consider the sequence given

$$
x_{n+1}=T x_{n}, \quad n=0,1,2, \ldots
$$

If there exists $n_{0} \in \mathbb{N}$ such that $x_{n_{0}+1}=x_{n_{0}}$, then the proof is completed. In this case, $x_{n_{0}}$ is the required fixed point of $T$. Throughout the proof, we assume that

$$
x_{n+1} \neq x_{n} \quad \text { for any } n=0,1,2, \ldots
$$

Notice that for any $n>0$ there exists $i_{n} \in\{1,2, \ldots, m\}$ such that $x_{n-1} \in A_{i_{n}}$ and $x_{n} \in A_{i_{n+1}}$, since $X=\bigcup_{i=1}^{m} A_{i}$. Due to the fact that $T$ is a cyclic $(\phi)$-contraction, we have

$$
p\left(x_{n}, x_{n+1}\right)=p\left(T x_{n-1}, T x_{n}\right) \leq \phi\left(p\left(x_{n-1}, x_{n}\right)\right),
$$

by taking $x=x_{n}$ and $y=x_{n+1}$ in (3). From (8) and taking the monotonicity of $\phi$ into account, we derive by induction that

$$
p\left(x_{n}, x_{n+1}\right) \leq \phi^{n}\left(p\left(x_{0}, x_{1}\right)\right) \quad \text { for any } n=1,2, \ldots
$$

As $p$ is an $E$-distance, we obtain that

$$
p\left(x_{n}, x_{m}\right) \leq p\left(x_{n}, x_{n+1}\right)+\cdots+p\left(x_{m-1}, x_{m}\right),
$$

so for $q \geq 1$ we have that

$$
p\left(x_{n}, x_{n+q}\right) \leq \phi^{n}\left(p\left(x_{0}, x_{1}\right)\right)+\cdots+\phi^{n+q-1}\left(p\left(x_{0}, x_{1}\right)\right) \text {. }
$$

In the sequel, we will prove that $\left\{x_{n}\right\}$ is a $p$-Cauchy sequence. Denoting

$$
S_{n}=\sum_{k=0}^{n} \phi^{k}\left(p\left(x_{0}, x_{1}\right)\right), \quad n \geq 0
$$

implies that

$$
p\left(x_{n}, x_{n+q}\right) \leq S_{n+q-1}-S_{n-1} \text {. }
$$

As $\phi$ is a $(c)$-comparison function, supposing $p\left(x_{0}, x_{1}\right)>0$, by Lemma 10, (iv), it follows that

$$
\sum_{k=0}^{\infty} \phi^{k}\left(p\left(x_{0}, x_{1}\right)\right)<\infty,
$$

so there is $S \in[0, \infty)$ such that

$$
\lim _{n \rightarrow \infty} S_{n}=S
$$


Then, by (13) we obtain that

$$
\lim _{n \rightarrow \infty} p\left(x_{n}, x_{n+q}\right)=0 \text {. }
$$

By repeating the same arguments in the proof of (16), we conclude that

$$
\lim _{n \rightarrow \infty} p\left(x_{n+q}, x_{n}\right)=0
$$

Consequently, we get that the sequence $\left\{x_{n}\right\}_{n \geq 0}$ is a $p$ Cauchy in the $S$-complete space $X=\bigcup_{i=1}^{m} A_{i}$. Thus, there exists $x \in X$ such that $\lim _{n \rightarrow \infty} x_{n}=x$. In what follows we prove that $x$ is a fixed point of $T$. In fact, since $\lim _{n \rightarrow \infty} x_{n}=x$, as $X=\bigcup_{i=1}^{m} A_{i}$ is a cyclic representation of $X$ with respect to $T$, the sequence $\left\{x_{n}\right\}$ has infinite terms in each $A_{i}$ for $i \in\{1,2, \ldots, m\}$.

Since $A_{i}$ is closed for every $i$, it follows that $x \in \bigcap_{i=1}^{m} A_{i}$; thus we take a subsequence $x_{n_{k}}$ of $\left\{x_{n}\right\}$ with $x_{n_{k}} \in A_{i-1}$. Using the contractive condition, we can obtain

$$
\begin{aligned}
p(x, T x) & \leq p\left(x, x_{n_{k+1}}\right)+p\left(x_{n_{k+1}}, T x\right) \\
& =p\left(x, x_{n_{k+1}}\right)+p\left(T x_{n_{k}}, T x\right) \\
& \leq p\left(x, x_{n_{k+1}}\right)+\phi\left(p\left(x_{n_{k}}, x\right)\right)
\end{aligned}
$$

and since $x_{n_{k}} \rightarrow x$ and $\phi$ belong to $\mathscr{C}$, letting $k \rightarrow \infty$ in the last inequality, we have $p(x, T x)=0$. Analogously, we can derive that $p(x, x)=0$ and, therefore, $x$ is a fixed point of $T$. This finishes the proof.

Corollary 16. Let $(X, \vartheta)$ be an $S$-complete Hausdorff uniform space such that $p$ is an E-distance on $X, m$ a positive integer, $A_{1}, A_{2}, \ldots, A_{m}$ nonempty closed subsets of $X$ with respect to the topological space $(X, \tau(\vartheta))$, and $X=\bigcup_{i=1}^{m} A_{i}$. Let operator $T: X \rightarrow X$ satisfy

(i) $p(T x, T y) \leq k p(x, y)$, for any $x \in A_{i}, y \in A_{i+1}, i=$ $1,2, \ldots, m$, where $A_{m+1}=A_{1}$ and $0<k<1$.

Then, $T$ has a unique fixed point $z \in \bigcap_{i=1}^{m} A_{i}$.

Proof. By Theorem 15, it is enough to set $\phi(t)=k t$.

Corollary 17 (cf. [16]). Let $(X, d)$ be a complete metric space, $m$ a positive integer, $A_{1}, A_{2}, \ldots, A_{m}$ nonempty closed subsets of $X$, and $X=\bigcup_{i=1}^{m} A_{i}$. Let $T: X \rightarrow X$ be a cyclic $(\phi)$ contraction. Then, $T$ has a unique fixed point $z \in \bigcap_{i=1}^{m} A_{i}$.

Proof. By Theorem 15, it is enough to set $\vartheta=\left\{U_{\epsilon} \mid \epsilon>0\right\}$.

Corollary 18 (cf. [13]). Let $(X, d)$ be a complete metric space, $m$ a positive integer, $A_{1}, A_{2}, \ldots, A_{m}$ nonempty closed subsets of $X$, and $X=\bigcup_{i=1}^{m} A_{i}$ a cyclic representation of $X$ with respect to $T$. Let $T: X \rightarrow X$ satisfy

$$
p(T x, T y) \leq k p(x, y)
$$

for any $x \in A_{i}, y \in A_{i+1}, i=1,2, \ldots, m$, where $k \in(0,1)$ and $A_{m+1}=A_{1}$. Then, $T$ has a unique fixed point $z \in \bigcap_{i=1}^{m} A_{i}$.
Definition 19. Let $(X, \vartheta)$ be a uniform space, $m$ a positive integer, $A_{1}, A_{2}, \ldots, A_{m}$ nonempty subsets of $X$, and $T, g$ : $X \rightarrow X$ self-mappings. An operator $T$ is a cyclic $(\phi)-g$ contraction if

(i) $g X=\bigcup_{i=1}^{m} g A_{i}$ is a cyclic representation of $X$ with respect to $T$,

(ii) $p(T x, T y) \leq \phi(p(g x, g y))$, for any $x \in A_{i}, y \in A_{i+1}$, $i=1,2, \ldots, m$, where $A_{m+1}=A_{1}$ and $\phi \in \mathscr{C}$.

Inspired by [28], we now prove a common fixed point theorem as an application of our Theorem 15.

Theorem 20. Let $(X, \vartheta)$ be a uniform space, $T, g: X \rightarrow X$ self-maps such that $T$ is cyclic $(\phi)$-g-contraction, and $g X S$ complete Hausdorff uniform space together with $p$ being an $E$ distance on $X$. Suppose that $g A_{1}, g A_{2}, \ldots, g A_{m}$ are nonempty closed subsets of $g X$ with respect to the uniform topology and TX $\subset g X=\bigcup_{i=1}^{m} g A_{i}$. Then, $T$ and $g$ have $a$ unique coincidence point. Moreover, if $T$ and $g$ are weakly compatible, then they have a unique common fixed point $z \in \bigcap_{i=1}^{m} g A_{i}$.

Proof. As $g: X \rightarrow X$, so there exists $E \subset X$ such that $g E=$ $g X$ and $g: E \rightarrow X$ is one-to-one. Now, since $T X \subset g X$, we define mappings $h: g E \rightarrow g E$ by $h(g x)=T x$. Since $g$ is one-to-one on $E$, so $h$ is well defined. As $T$ is cyclic $(\phi)-g$ contraction, so

$$
p(T x, T y) \leq \phi(p(g x, g y))
$$

for any $g x \in g A_{i}, g y \in g A_{i+1}, i=1,2, \ldots, m$. Thus,

$$
p(h(g x), h(g y))=p(T x, T y) \leq \phi(p(g x, g y))
$$

for any $g x \in g A_{i}, g y \in g A_{i+1}, i=1,2, \ldots, m$, which implies that $h$ is cyclic $(\phi)$-contraction on $g X$. Hence, all the conditions of Theorem 15 are satisfied by $h$, so $h$ has a unique fixed point $z=g x$ in $g X$. That is, $g x=z=h(z)=h(g x)=$ $T x$, so $T$ and $g$ have a unique coincidence point as required. Moreover, if $T$ and $g$ are weakly compatible, then they have a unique common fixed point.

Corollary 21 (cf. Theorem $3.2[18]$ ). Let $(X, 9)$ be a uniform space, $T, g: X \rightarrow X$ self-maps such that $T$ is $(\phi)$ $g$-contraction, and $g X S$-complete Hausdorff uniform space together with $p$ being an E-distance on $X$. Suppose that TX $\subset$ $g X$ and $T$ and $g$ are commuting. Then, $T$ and $g$ have $a$ unique common fixed point $z \in X$.

Proof. Take $A_{i}=X$ for all $i=1, \ldots, m$ in Theorem 20 .

Example 22. Let $(X, d)$ be a metric space, where $X=\{1 / n\} \cup$ $\{0\}$ and $d=|$,$| . Set A_{1}=\{1 / 3 n\} \cup\{0,1\}, A_{2}=\{1 /(3 n+1)\} \cup$ $\{0,1\}$, and $A_{3}=\{1 /(3 n+2)\} \cup\{0,1\}$. Define $\vartheta=\left\{U_{\epsilon} \mid \epsilon>0\right\}$. It is easy to see that $(X, \vartheta)$ is a uniform space. If we define $\phi$ : $[0, \infty) \rightarrow[0, \infty)$ by $\phi(t)=k t$ for $0<k<1$ and $T: X \rightarrow X$ 
by $T(0)=T(1)=0$ and $T(1 / n)=1 /(4 n+1)$, then for every $x, y \neq 0,1$ we have

$$
\begin{aligned}
d(T x, T y) & =d\left(\frac{1}{4 n+1}, \frac{1}{4 m+1}\right) \\
& =\frac{|4(m-n)|}{|4 n+1||4 m+1|} \leq \frac{|m-n|}{4 n \cdot m} \\
& \leq \frac{1}{4}\left|\frac{1}{n}-\frac{1}{m}\right|=\frac{1}{4} d(x, y) .
\end{aligned}
$$

Also, for $x, y=0,1$ the above inequality obviously holds. This shows that the contractive condition of Corollary 16 is satisfied and 0 is fixed point $T$.

Definition 23. Let $(X, \vartheta)$ be a uniform space, let $f, g: X \rightarrow$ $X$ be two mappings, and let $A$ and $B$ be nonempty closed subsets of $X$. The $X=A \cup B$ is said to be a cyclic representation of $X$ with respect to the pair $(f, g)$ if $f(A) \subset B$ and $g(B) \subset A$.

Definition 24. Let $(X, \vartheta)$ be a uniform space, $A, B$ nonempty subsets of $X$, and $X=A \cup B$. Two self-maps $f, g: X \rightarrow X$ are called cyclic $(\phi)$-contraction pair if

(i) $X=A \cup B$ is a cyclic representation of $X$ with respect to the pair $(f, g)$,

(ii) $\max \{p(f x, g y), p(g y, f x)\}$ $\phi(\max \{p(x, f x), p(y, g y)\})$, for any $x \in A, y \in B$, where $\phi \in \mathscr{C}$.

Theorem 25. Let $(X, 9)$ be an S-complete Hausdorff uniform space such that $p$ is an E-distance on $X$ and let $A, B$ be nonempty closed subsets of $X$ with respect to the topological space $(X, \tau(\vartheta))$ and $X=A \cup B$. Suppose that $f, g: X \rightarrow X$ are cyclic $(\phi)$-contraction pair. Then, $f$ and $g$ have a unique common fixed point $x \in A \cap B$.

Proof. Take $x_{0} \in X$ and consider the sequence given by

$$
f x_{2 n}=x_{2 n+1}, \quad g x_{2 n+1}=x_{2 n+2}, \quad n=0,1,2, \ldots
$$

Since $X=A \cup B$, for any $n>0, x_{2 n} \in A$, and $x_{2 n+1} \in B$, and $(f, g)$ are cyclic $(\phi)$-contraction pair, we have

$$
\begin{aligned}
p\left(x_{2 n+1}, x_{2 n+2}\right) & =p\left(f x_{2 n}, g x_{2 n+1}\right) \\
& \leq \max \left\{p\left(f x_{2 n}, g x_{2 n+1}\right), p\left(g x_{2 n+1}, f x_{2 n}\right)\right\} \\
& \leq \phi\left(\max \left\{p\left(x_{2 n}, f x_{2 n}\right), p\left(x_{2 n+1}, g x_{2 n+1}\right)\right\}\right) \\
& =\phi\left(\max \left\{p\left(x_{2 n}, x_{2 n+1}\right), p\left(x_{2 n+1}, x_{2 n+2}\right)\right\}\right) .
\end{aligned}
$$

Hence,

$$
p\left(x_{2 n+1}, x_{2 n+2}\right) \leq \phi\left(p\left(x_{2 n}, x_{2 n+1}\right)\right) \text {. }
$$

Similarly, we have

$$
\begin{aligned}
p\left(x_{2 n}, x_{2 n+1}\right) & =p\left(g x_{2 n-1}, f x_{2 n}\right) \\
& \leq \max \left\{p\left(f x_{2 n}, g x_{2 n-1}\right), p\left(g x_{2 n-1}, f x_{2 n}\right)\right\} \\
& \leq \phi\left(\max \left\{p\left(x_{2 n}, x_{2 n+1}\right), p\left(x_{2 n-1}, x_{2 n}\right)\right\}\right) .
\end{aligned}
$$

Hence,

$$
p\left(x_{2 n}, x_{2 n+1}\right) \leq \phi\left(p\left(x_{2 n-1}, x_{2 n}\right)\right) .
$$

From inequalities (25) and (27) and taking into account the monotonicity of $\phi$, we get by induction that

$$
p\left(x_{n}, x_{n+1}\right) \leq \phi^{n}\left(p\left(x_{0}, x_{1}\right)\right) \quad \text { for any } n=1,2, \ldots
$$

Since $p$ is an $E$-distance, we find that

$$
p\left(x_{n}, x_{m}\right) \leq p\left(x_{n}, x_{n+1}\right)+\cdots+p\left(x_{m-1}, x_{m}\right),
$$

so for $q \geq 1$ we have that

$$
p\left(x_{n}, x_{n+q}\right) \leq \phi^{n}\left(p\left(x_{0}, x_{1}\right)\right)+\cdots+\phi^{n+q-1}\left(p\left(x_{0}, x_{1}\right)\right) \text {. }
$$

In the sequel, we will prove that $\left\{x_{n}\right\}$ is a $p$-Cauchy sequence. Denote

$$
S_{n}=\sum_{k=0}^{n} \phi^{k}\left(p\left(x_{0}, x_{1}\right)\right), \quad n \geq 0 .
$$

By relation (31), we have

$$
p\left(x_{n}, x_{n+q}\right) \leq S_{n+q-1}-S_{n-1} .
$$

Regarding $\phi \in \mathscr{C}$ together with Lemma 10(iv), we get that

$$
\sum_{k=0}^{\infty} \phi^{k}\left(p\left(x_{0}, x_{1}\right)\right)<\infty,
$$

since $p\left(x_{0}, x_{1}\right)>0$. Thus, there is $S \in[0, \infty)$ such that

$$
\lim _{n \rightarrow \infty} S_{n}=S \text {. }
$$

Then, by (32) we obtain that

$$
p\left(x_{n}, x_{n+q}\right) \longrightarrow 0 \text { as } n \longrightarrow \infty \text {. }
$$

In an analogous way, we derive that

$$
p\left(x_{n+q}, x_{n}\right) \longrightarrow 0 \text { as } n \longrightarrow \infty \text {. }
$$

Hence, we get that $\left\{x_{n}\right\}_{n \geq 0}$ is a $p$-Cauchy sequence in the $S$-complete space $X=A \cup B$. So there exists $x \in X$ such that $\lim _{n \rightarrow \infty} f x_{2 n}=\lim _{n \rightarrow \infty} g x_{2 n+1}=x$. In what follows, we prove that $x$ is a fixed point of $f, g$. In fact, since $\lim _{n \rightarrow \infty} f x_{2 n}=\lim _{n \rightarrow \infty} g x_{2 n+1}=x$ and as $X=A \cup B$ is a 
cyclic representation of $X$ with respect to $f, g$, the sequence $\left\{x_{n}\right\}$ has infinite terms in each $A, B$.

Since $A, B$ are closed, it follows that $x \in A \cap B$; thus we take subsequences $x_{2 n}, x_{2 n+1}$ of $\left\{x_{n}\right\}$ with $x_{2 n} \in A$ and $x_{2 n+1} \in$ $B$. Using the contractive condition, we can obtain

$$
\begin{aligned}
& p(x, f x) \leq p\left(x, x_{2 n+2}\right)+p\left(x_{2 n+2}, f x\right) \\
&=p\left(x, x_{2 n+2}\right)+p\left(g x_{2 n+1}, f x\right) \\
& \leq p\left(x, x_{2 n+2}\right)+\max \left\{p\left(g x_{2 n+1}, f x\right),\right. \\
&\left.p\left(f x, g x_{2 n+1}\right)\right\} \\
& \leq p\left(x, x_{2 n+2}\right)+\phi(\max \{p(x, f x), \\
&\left.\left.p\left(x_{2 n+1}, x_{2 n+2}\right)\right\}\right),
\end{aligned}
$$

and since $x_{n} \rightarrow x$ and $\phi$ belong to $\mathscr{C}$, letting $n \rightarrow \infty$ in the last inequality, we have $p(x, f x) \leq \phi(p(x, f x))<p(x, f x)$; hence $p(x, f x)=0$. Similarly, we can show that $p(x, x)=0$ and, therefore, $x$ is a fixed point of $f$. Similarly, we can show that $x$ is a fixed point of $g$. Finally, in order to prove the uniqueness of the fixed point, we have $y, z \in X$ with $y$ and $z$ fixed points of $f, g$. The cyclic character of $f, g$ and the fact that $y, z \in X$ are fixed points of $f, g$ imply that $y, z \in A \cap B$. Using the contractive condition, we obtain

$$
\begin{aligned}
p(y, z)=p(f y, g z) & \leq \max \{p(f y, g z), p(g z, f y)\} \\
& \leq \phi(\max \{p(y, f y), p(z, g z)\})=0,
\end{aligned}
$$

and from the last inequality we get

$$
p(y, z)=0 .
$$

Using the same arguments above, we can show that $p(y, y)=$ 0 and, consequently, $y=z$. This finishes the proof.

Corollary 26. Let $(X, d)$ be a complete metric space and $A, B$ nonempty closed subsets of $X$ and $X=A \cup B$. Let $f, g: X \rightarrow$ $X$ be cyclic $(\phi)$-contraction pair. Then, $f$ and $g$ have a unique common fixed point $z \in A \cap B$.

Proof. By Theorem 25, it is enough to set $\vartheta=\left\{U_{\epsilon} \mid \epsilon>0\right\}$.

Corollary 27. Let $(X, \vartheta)$ be an $S$-complete Hausdorff uniform space such that $p$ is an E-distance on $X$ and let $A, B$ be nonempty closed subsets of $X$ with respect to the topological space $(X, \tau(\vartheta))$ and $X=A \cup B$. Suppose that the maps $f, g$ : $X \rightarrow X$ satisfy the following inequality:

(i) $\max \{p(f x, g y), p(g y, f x)\}$ $k \max \{p(x, f x), p(y, g y)\}$, for any $x \in A, y \in B$, where $0<k<1$.

Then, $f, g$ have a unique common fixed point $z \in A \cap B$.

Proof. By Theorem 25, it is enough to set $\phi(t)=k t$.
Example 28. Let $(X, p)$ be a partial metric space, where $X=$ $\{1 / n\} \cup\{0,1\}$ and $p(x, y)=\max \{x, y\}$. Set $A=\{1 / 2 n\} \cup\{0,1\}$ and $B=\{1 /(2 n+1)\} \cup\{0,1\}$. Define $\vartheta=\left\{U_{\epsilon} \mid \epsilon>0\right\}$, where $U_{\epsilon}=\left\{(x, y) \in X^{2}: p(x, y)<p(x, x)+\epsilon\right\}$. It is easy to see that $(X, \vartheta)$ is a uniform space. If we define $\phi:[0, \infty) \rightarrow[0, \infty)$ by $\phi(t)=k t$ for $0<k<1$ and $f: X \rightarrow X$ by $f(1 / 2 n)=$ $1 /(4 n+1), f(0)=f(1)=0$, and $g(1 /(2 n+1))=1 /(4 n+2)$, $g(0)=g(1)=0$. Then, for every $x, y \neq 0,1$ we have

$$
\begin{aligned}
\max & \{p(f x, g y), p(g y, f x)\} \\
= & \max \left\{p\left(\frac{1}{4 n+1}, \frac{1}{4 m+2}\right)\right. \\
& \left.p\left(\frac{1}{4 m+2}, \frac{1}{4 n+1}\right)\right\} \\
= & \max \left\{\frac{1}{4 n+1}, \frac{1}{4 m+2}\right\} \\
\leq & \frac{1}{2} \max \left\{\frac{1}{2 n}, \frac{1}{2 m+1}\right\} \\
= & \frac{1}{2} \max \{p(x, f x), p(y, g y)\}
\end{aligned}
$$

for any $x \in A, y \in B$. Also, for $x, y=0,1$ the above inequality obviously holds. This shows that the contractive condition of Corollary 27 is satisfied and 0 is a common fixed point of $f$ and $g$.

\section{Conflict of Interests}

The authors declare that there is no conflict of interests regarding the publication of this paper.

\section{Acknowledgment}

This paper was funded by the Deanship of Scientific Research (DSR), King Abdulaziz University, Jeddah. Therefore, the first author acknowledges with thanks DSR, KAU, for the financial support.

\section{References}

[1] N. Bourbaki, Éléments de mathématique. Fasc. II. Livre III: Topologie générale. Chapitre 1: Structures Topologiques. Chapitre 2: Structures Uniformes, vol. 1142 of Actualités Scientifiques et Industrielles, Hermann, Paris, France, 1965.

[2] E. Zeidler, Nonlinear Functional Analysis and its Applications, vol. 1, Springer, New York, NY, USA, 1986.

[3] V. Berinde, Iterative Approximation of Fixed Points, Springer, Berlin, Germany, 2007.

[4] J. Jachymski, "Fixed point theorems for expansive mappings," Mathematica Japonica, vol. 42, no. 1, pp. 131-136, 1995.

[5] O. Kada, T. Suzuki, and W. Takahashi, "Nonconvex minimization theorems and fixed point theorems in complete metric spaces," Mathematica Japonica, vol. 44, no. 2, pp. 381-391, 1996.

[6] M. Cherichi and B. Samet, "Fixed point theorems on ordered gauge spaces with applications to nonlinear integral equations," Fixed Point Theory and Applications, vol. 2012, article 13, 2012. 
[7] M. O. Olatinwo, "Some common fixed point theorems for selfmappings in uniform space," Acta Mathematica, vol. 23, no. 1, pp. 47-54, 2007.

[8] I. Altun and M. Imdad, "Some fixed point theorems on ordered uniform spaces," Filomat, vol. 23, pp. 15-22, 2009.

[9] E. Tarafdar, "An approach to fixed-point theorems on uniform spaces," Transactions of the American Mathematical Society, vol. 191, pp. 209-225, 1974.

[10] B. E. Rhoades, "A comparison of various definitions of contractive mappings," Transactions of the American Mathematical Society, vol. 226, pp. 257-290, 1977.

[11] I. A. Rus, Generalized Contractions and Applications, Cluj University Press, Cluj-Napoca, Romania, 2001.

[12] S. Z. Wang, B. Y. Li, Z. M. Gao, and K. Iséki, "Some fixed point theorems on expansion mappings," Mathematica Japonica, vol. 29, no. 4, pp. 631-636, 1984.

[13] W. A. Kirk, P. S. Srinivasan, and P. Veeramani, "Fixed points for mappings satisfying cyclical contractive conditions," Fixed Point Theory, vol. 4, no. 1, pp. 79-89, 2003.

[14] E. Karapınar and K. Sadarangani, "Fixed point theory for cyclic $(\phi-\psi)$-contractions," Fixed Point Theory and Applications, vol. 2011, article 69, 2011.

[15] E. Karapınar and H. K. Nashine, "Fixed point theorem for cyclic Chatterjea type contractions," Journal of Applied Mathematics, vol. 2012, Article ID 165698, 15 pages, 2012.

[16] M. Păcurar and I. A. Rus, "Fixed point theory for cyclic $\varphi$ contractions," Nonlinear Analysis: Theory, Methods \& Applications, vol. 72, no. 3-4, pp. 1181-1187, 2010.

[17] I. A. Rus, "Cyclic representations and fixed points," Annals of the Tiberiu Popoviciu Seminar of Functional Equations, Approximation and Convexity, vol. 3, pp. 171-178, 2005.

[18] M. Aamri and D. El Moutawakil, "Common fixed point theorems for $E$-contractive or $E$-expansive maps in uniform spaces," Acta Mathematica, vol. 20, no. 1, pp. 83-91, 2004.

[19] M. Aamri and D. El Moutawakil, "Weak compatibility and common fixed point theorems for A-contractive and E-expansive maps in uniform spaces," Serdica, vol. 31, no. 1-2, pp. 75-86, 2005.

[20] M. Aamri, S. Bennani, and D. El Moutawakil, "Fixed points and variational principle in uniform spaces," Siberian Electronic Mathematical Reports, vol. 3, pp. 137-142, 2006.

[21] R. P. Agarwal, D. O’Regan, and N. S. Papageorgiou, “Common fixed point theory for multivalued contractive maps of Reich type in uniform spaces," Applicable Analysis, vol. 83, no. 1, pp. 37-47, 2004.

[22] V. Berinde, Contractii Generalizate si Aplicatii, vol. 22, Editura Cub Press, Baia Mare, Romania, 1997.

[23] V. Popa, "A general fixed point theorem for two pairs of mappings on two metric spaces," Novi Sad Journal of Mathematics, vol. 35, no. 2, pp. 79-83, 2005.

[24] N. Hussain, Z. Kadelburg, S. Radenović, and F. Al-Solamy, "Comparison functions and fixed point results in partial metric spaces," Abstract and Applied Analysis, vol. 2012, Article ID 605781, 15 pages, 2012.

[25] N. Hussain and H. K. Pathak, "Common fixed point and approximation results for $H$-operator pair with applications," Applied Mathematics and Computation, vol. 218, no. 22, pp. 11217-11225, 2012.

[26] N. Hussain, G. Jungck, and M. A. Khamsi, "Nonexpansive retracts and weak compatible pairs in metric spaces," Fixed Point Theory and Applications, vol. 2012, article 100, 2012.
[27] E. Karapinar, "Fixed point theory for cyclic weak $\phi$ contraction," Applied Mathematics Letters, vol. 24, no. 6, pp. 822-825, 2011.

[28] R. H. Haghi, Sh. Rezapour, and N. Shahzad, "Some fixed point generalizations are not real generalizations," Nonlinear Analysis: Theory, Methods \& Applications, vol. 74, no. 5, pp. 1799-1803, 2011. 


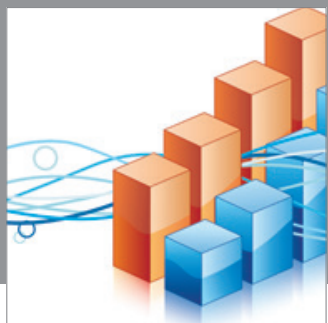

Advances in

Operations Research

mansans

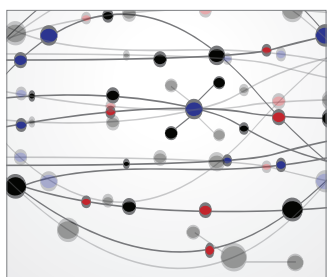

The Scientific World Journal
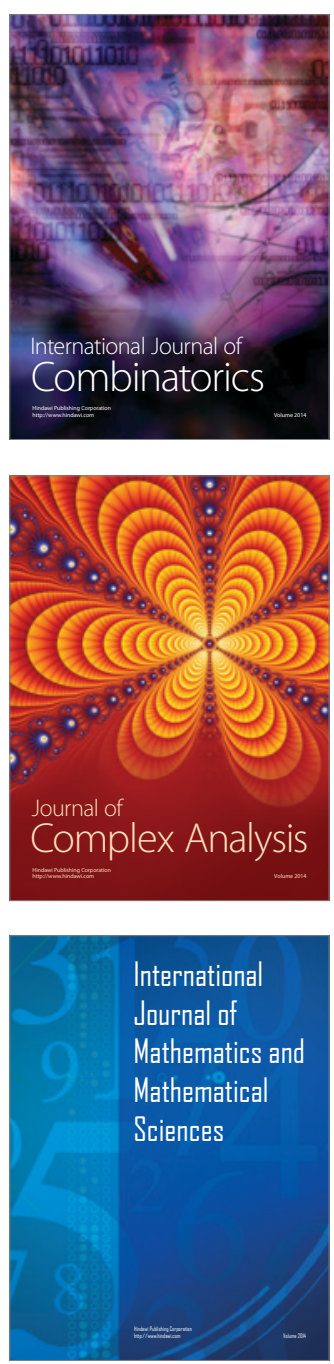
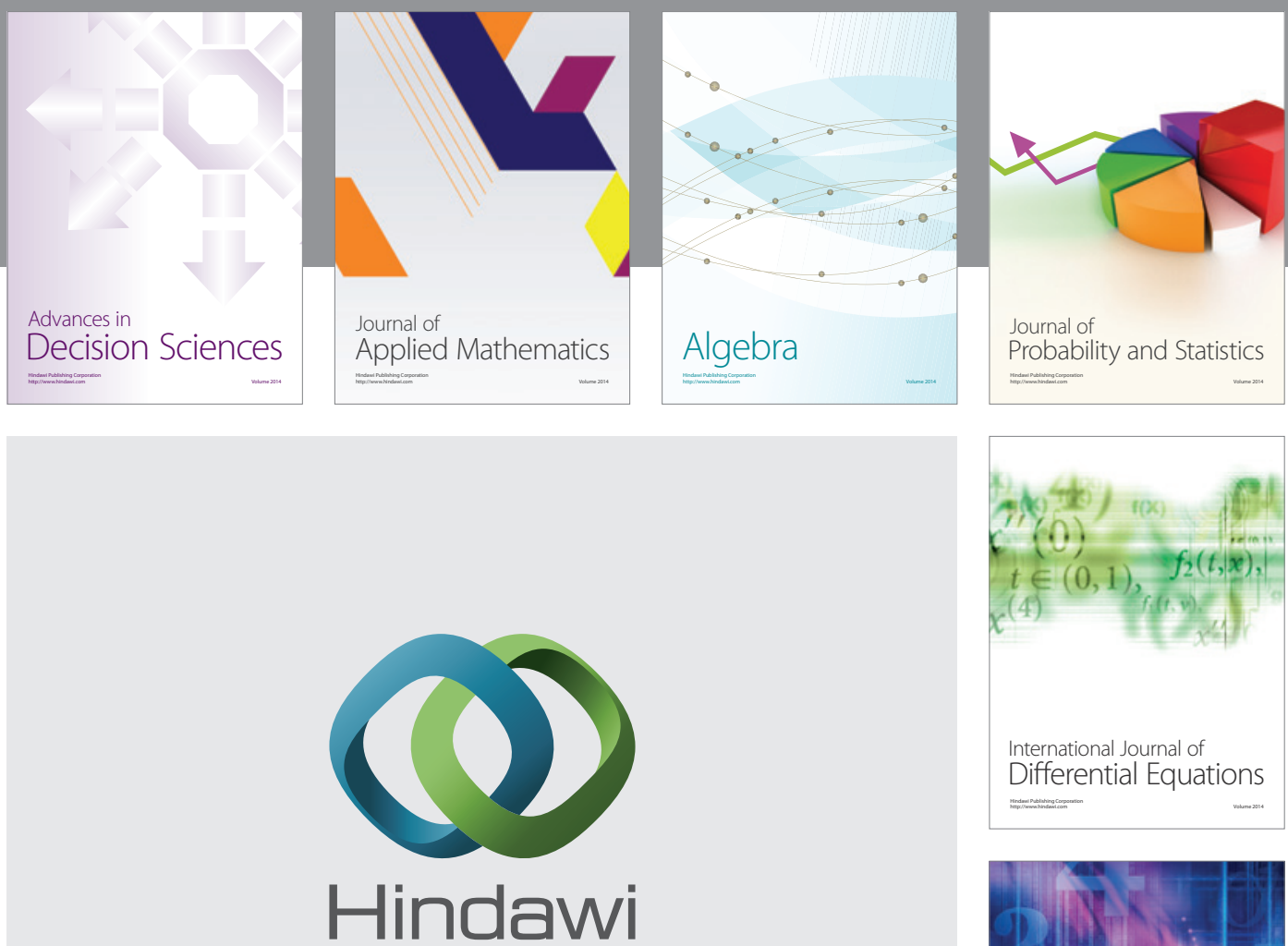

Submit your manuscripts at http://www.hindawi.com
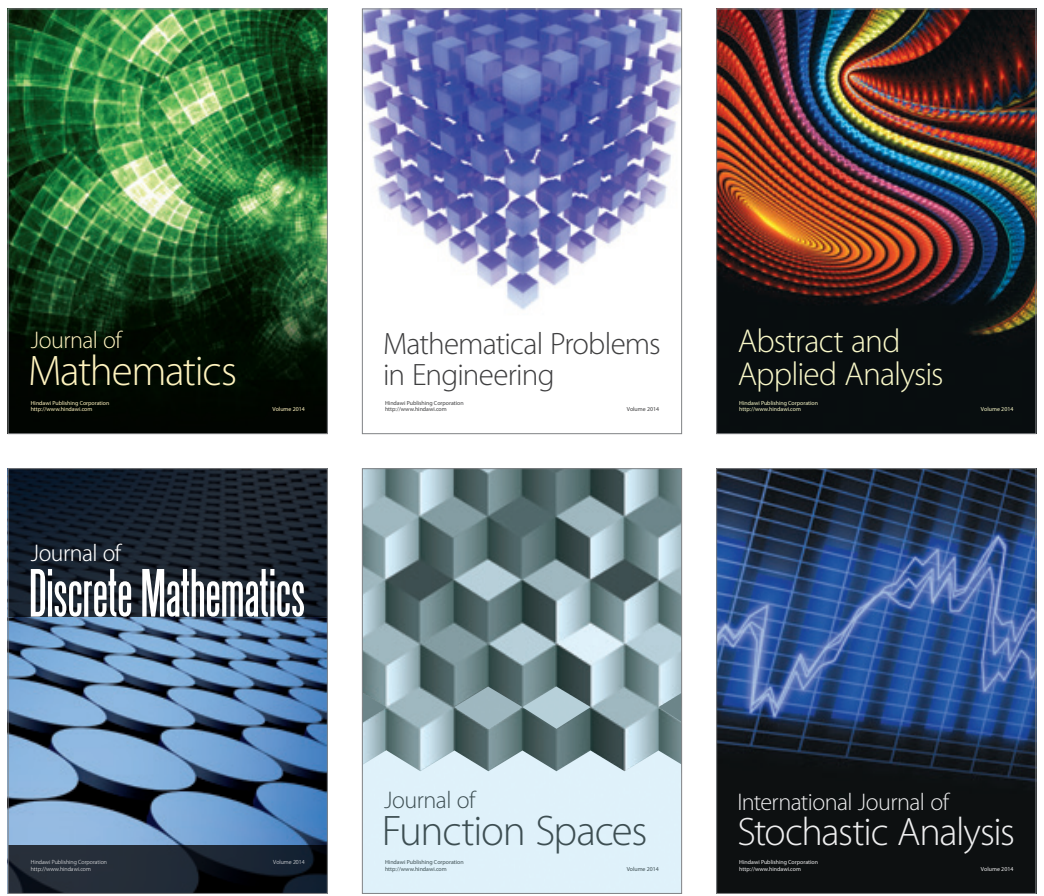

Journal of

Function Spaces

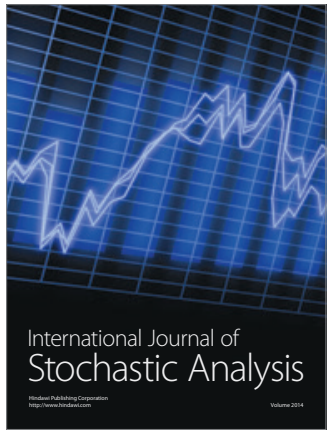

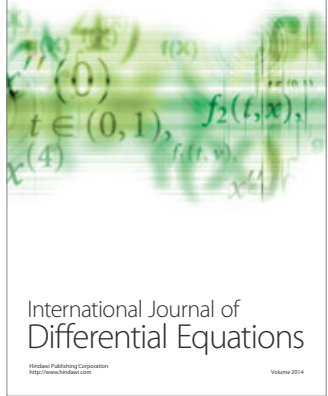
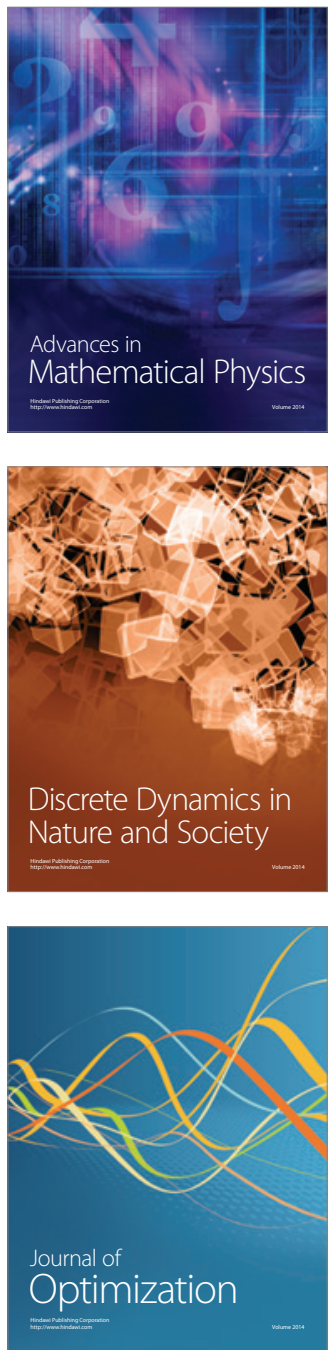